\title{
Research on Mine Tunnel Positioning Technology based on the Oblique Triangle Layout Strategy
}

\author{
Jingzhao Li*, Hong Ma and Yubo Cui \\ School of Computer Science and Engineering, Anhui University of Science and Technology, Huainan, China
}

Received: 4 Jun. 2013, Revised: 30 Aug. 2013, Accepted: 2 Sep. 2013

Published online: 1 Jan. 2014

\begin{abstract}
In allusion to the special environment of coal mine roadways, the strategy laying anchor nodes evenly on the left and right sides and the top of roadways in accordance with the oblique triangle and the mine personnel positioning algorithm based on received and transmitted signal strength of nodes were adopted in the paper. The algorithm estimating the distance between an unknown node and an anchor node through built-in received and transmitted signal strength information of the anchor node could realize the mine personnel positioning with only two anchor nodes. Both theoretical analysis and simulation experiments show that it improves the personnel positioning accuracy in the coal mine roadway environment effectively used the effective combination of the nodes arrangement strategy and the positioning algorithm proposed in the paper.
\end{abstract}

Keywords: anchor nodes, signal strength, roadway, oblique triangle, positioning

\section{Introduction}

Our country is a large mineral resources production and consumption country and the development of the national economy depends heavily on mineral resources. For the coal mine environment which is complex and special and different from the outdoor environment, the personnel positioning in such an environment is affected by many factors, such as the temperature of the coal mine, air humidity and dust [1], etc. In the age of the Internet, when the coal mine news is searched optionally, mining accidents are often displayed [2]. So it is no doubt about the importance and necessity of research for coal mine. In this paper, in view of the particularity of mine roadway environment, the method to emplace anchor nodes on the top of roadways is given, through analyzing this special stripe space and in allusion to the method, the mine personnel positioning algorithm based on signal strength of nodes sending and receiving is provided. Without additional positioning equipment, to arrange anchor nodes early according to the method is only needed to do. Then the coordinates of the unknown node are got by the method proposed in the paper. Simulation experiments show that the positioning algorithm can get good positioning accuracy and also save costs.

\section{Coal Mine Wireless Signal Model and Anchor Nodes Laying Strategy}

\subsection{The Foundation of Coal Mine Wireless Signal Model}

According to different positioning mechanisms, the wireless sensor network localization algorithms are divided into two main categories that the one is ranged-based and the other range-free [3]. The former includes typical algorithms, such as TOA [4], TDOA, AOA [5] and the RSSI, etc. and the latter includes typical algorithms, such as DV-Hop algorithm [6], convex programming algorithm and APIT algorithm. Although the latter has a comparative advantage versus the former in the power consumption and cost, there are many shortcomings in the positioning accuracy. Aiming at the mine personnel positioning [7], the method to make use of RSSI becomes a focus of study because of limitations of the space and the surrounding environment.

The coal mine wireless signal transmission model based on RSSI can be represented by the formula (1)

$$
\operatorname{RSSI}(\mathrm{dBm})=-(A+10 \cdot n \lg d)
$$

It can be seen from the formula (1) that the relationship between received signal strength and signal

\footnotetext{
*Corresponding author e-mail: lijinzhao097@gmail.com
} 
transmission distance is determined by the values of the constant $A$ and $n$. RF parameters $A$ and $n$ are used to describe the network operating environment. The RF parameter $A$, which is expressed in $\mathrm{dBm}$, is defined as the absolute value of average received energy of the place where is one meter from the emitter. For example, when the average received energy is $-40 \mathrm{dBm}$, the parameter $A$ is set at 40 . The RF parameter $\mathrm{n}$ indicates the decaying rate of signal energy with the increase of the distance between the transmitter and the receiver.

There is a certain degree of error when RSSI is used for positioning. As the transmission distance is closer, the power decreases rapidly and the farther the transmission distance, the slower the attenuation [8]. After averaging the values obtained from several measurements, an equals to -46 and $n$ equals to 2.87 . With the change of the transmission distance, the change of signal power is not obvious as the transmission distance is close to about $60 \mathrm{~m}$. Therefore, in the RSSI-based location technology, the closer the transmission distance, the more accurate the positioning has and the farther the transmission distance, the larger the positioning error.

\subsection{The Laying Strategy of Anchor Nodes in the Coal Mine Roadway}

Coal mine roadway which generally takes on a strip with arch-section has several meters in width and height and hundreds of meters, even thousands of meters in length. For such a special shape of space, with a large error produced, those positioning algorithms suitable for open-air environment are limited. How to realize the personnel positioning in the coal mine roadway is seen as the main subject of the research. The personnel positioning is focused on its length direction (horizontal ordinate) because the width and the height of roadway are too small when compared with the length. In this topic, priority is given to how to lay anchor nodes to improve the communication range of anchor nodes, reduce the energy consumption of nodes and improve the personnel positioning accuracy.

The radio signal propagation in coal mine roadway is affected by cramped space, inhomogeneous geological structure and mineral powder and water vapor in the air. In order to reduce these effects, the emplacement of sensor nodes is extended to the top of the arch tunnel and the nodes layout is shown in Fig. 1.

In the roadway, anchor nodes are laid evenly on the left and right sides. The height of the roadway is assumed to be 3 meters and anchor nodes are laid at height of 1.5 meters in order to reduce the environment effect to the communication between nodes. Meanwhile sensor nodes personnel carried are also worn at heights of 1.5 meters, as shown in Fig. 1.

In Fig. 1, L1, L2... are anchor nodes laid on the left side of the roadway and $\mathrm{R} 1, \mathrm{R} 2 .$. , are anchor nodes laid

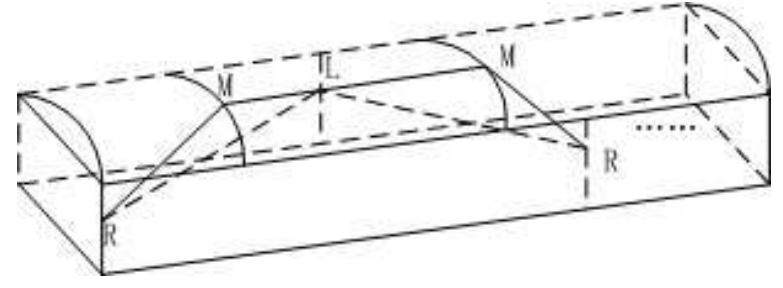

Fig. 1: Nodes layout of the coal mine underground roadway

on the left side and M1, M2, are anchor nodes laid on the top. In the process of positioning, the density of anchor nodes is determined by the communication range of the anchor nodes. ZigBee has a transmission range from tens of meters to hundreds of meters and the communication range of anchor nodes used in the experiments is $120 \mathrm{~m}$. Through the analysis above, it is concluded that a small error of RSSI is got when the distance is within $60 \mathrm{~m}$.In order to make full use of the communication range of the anchor nodes, anchor nodes are laid on both sides and the top of the roadway at 60 meters intervals. For the little gap in height between the nodes of both sides and the top anchor nodes, all the nodes can be seen as on one plane. The layout situation of all anchor nodes profiles is shown in Fig. 2.

\section{The Positioning Algorithm of Coal Mine Underground Roadway based on the RSSI}

\subsection{The Feasibility Analysis of the Two-Point Positioning Method}

The two-point positioning method is shown in Fig. 3. As known in Fig. 3, the rectangular area is a roadway $60 \mathrm{~m}$ by $5 \mathrm{~m}$ and two of the four intersections above are unknown nodes whose coordinates are 19.81, 3.138 and 50.02, 4.589. The abscissa, $(19.81+20.41) / 2=20.11$, is obtained for the upper-left node unknown by the two-point positioning method and the difference between the calculated and the actual abscissa is $|20.11-19.81|=0.3 ; \quad$ The abscissa, $(50.02+50.72) / 2=50.37$, is obtained for the upper-right node unknown by the two-point positioning method and the difference between the calculated and the actual abscissa is $|50.37-50.02|=0.35$. The small error in the ideal case shows that it is feasible to use the positioning algorithm. Whether the algorithm is valid will be determined by whether the distance achieved by the measured RSSI is close to the true distance between the unknown node and the anchor node.

\subsection{The Two-Point Positioning Method}

Through the received signal strength measured, signal strength ranging method makes use of the formula to 


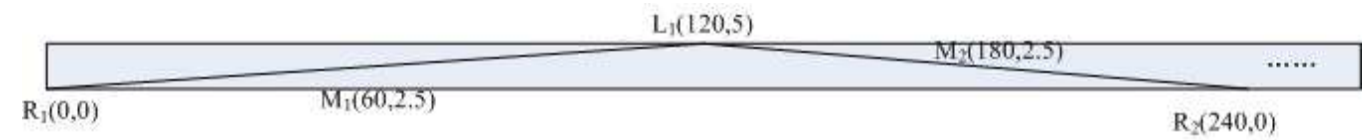

Fig. 2: The planar graph of anchor nodes layout

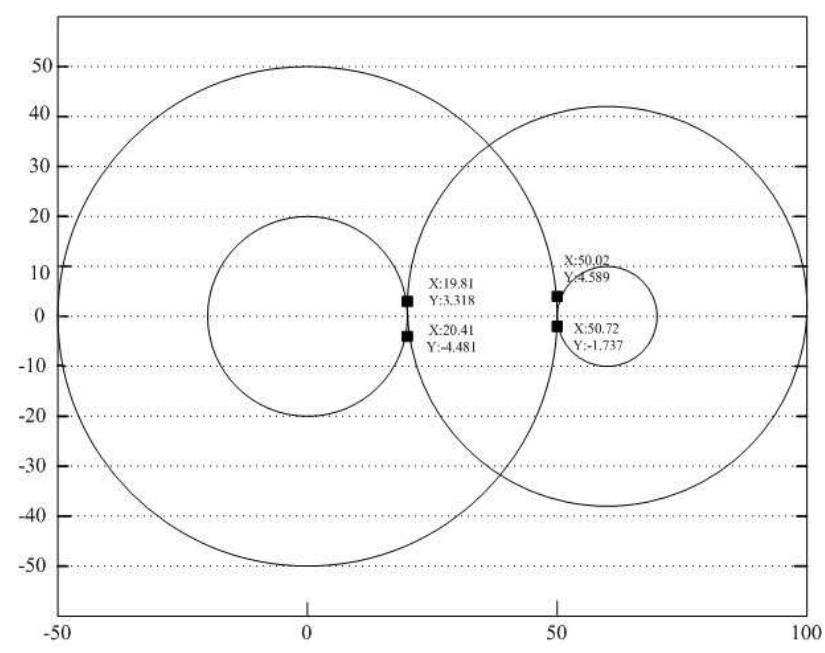

Fig. 3: The feasibility analysis of the two-point positioning method with averaging abscissas

calculated the approximate distance between the transmitting and receiving nodes, the distance between anchor nodes and unknown ones and then the location of unknown nodes can be obtained by trilateration or maximum relieved estimation method.

The information about distances between three or more anchor nodes and unknown nodes should be obtained for the trilateration or maximum relieved estimation method so that high density of anchor nodes should be required in the network, which increases the cost of the entire network. In view of this, the trilateration or maximum relieved estimation method is not used and the two-point positioning method is introduced.

The basic steps of the two-point positioning method are as follows:

Step one: anchor nodes are placed fixedly and unknown nodes randomly in the area Border_length in length and Border_width in width. The location of anchor nodes is known and the packets containing location information about $X$ axis and $Y$ axis from anchor nodes will be sent off to other unknown nodes.

Step two: the unknown nodes send requests off to the anchor nodes which can communicate with them, receive the response signals of the reference nodes and read out the received $R S S I$ value.

Step Three: each unknown node will calculate its own position by using hardware location engine and the value of $X$, the value of $Y$, RSSI of anchor nodes which communicate with them and the positioning engine is just a simple interface at the software level. When parameters are wrote into it, the calculated position information will be read.

Step four: the input parameters of positioning function of the interface at the software level are the value of $A$ and the value of $n$ in the coal mine roadway, the physical location $(X, Y)$ of the anchor nodes and the value of the RSSI that the unknown nodes receive from the anchor nodes [9]. The value of $A$ and the value of $n$ are achieved by repeated experiments in this environment.

Step Five: the coordinates of the anchor nodes communicating with the unknown node $(x, y)$ are assumed to be $\left(X_{1}, Y_{1}\right),\left(X_{2}, Y_{2}\right), \ldots\left(X_{m}, Y_{m}\right)$, as shown in Figure 4 . The unknown node is represented by a hollow circle and the anchor nodes are solid circles. Anchor nodes are located every 60 meters and for an unknown node, only two of them meet positioning requirements. The coordinates of the two anchor nodes are assumed to be $\left(x_{i}, y_{i}\right)$ and $\left(x_{j}, y_{j}\right)$ and the coordinate of the unknown node is $(x, y)$. Then the coordinates of the unknown node can be calculated by the formula (2).

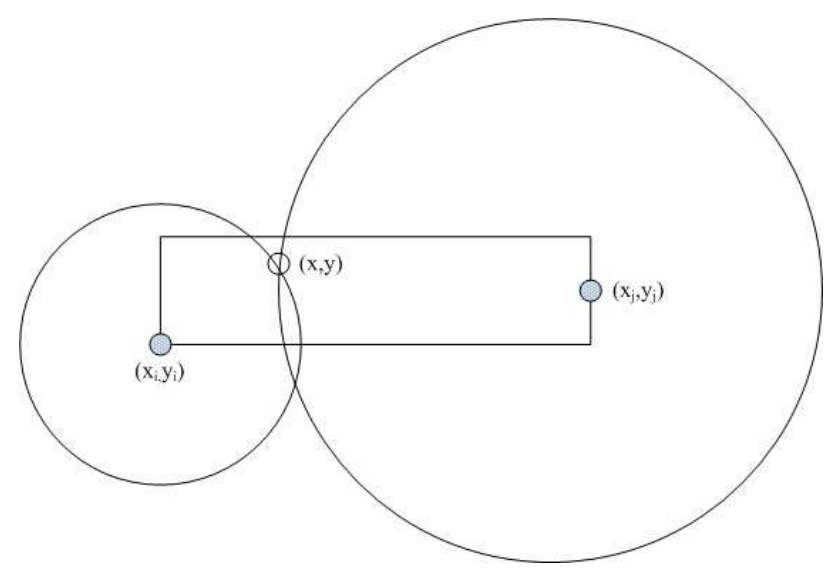

Fig. 4: The schematic diagram of the two-point positioning method

$$
\left\{\begin{array}{l}
\left(x_{i}-x\right)^{2}+\left(y_{i}-y\right)^{2}=d_{i}^{2} \\
\left(x_{j}-x\right)^{2}+\left(y_{j}-y\right)^{2}=d_{j}^{2}
\end{array}\right.
$$

The distances between the unknown node and the two anchor nodes $\left(x_{i}, y_{i}\right)$ and $\left(x_{j}, y_{j}\right)$ are represented by $d_{i}, d_{j}$ 
respectively and can be estimated by the formula (3).

$$
d=10^{-(R S S I+A) /(10 \times n)}
$$

In the theoretical case, two coordinates, $\left(x_{1}, y_{1}\right)$ and $\left(x_{2}, y_{2}\right)$, will be obtained by the formula (2) for any unknown node. It is unnecessary to get the ordinates of the unknown nodes [10] for the width of roadway which is generally less than $5 \mathrm{~m}$ and the abscissa of unknown node, $x=\left(x_{1}+x_{2}\right) / 2$, is achieved directly by taking the average of the two obtained abscissas.

\section{Analysis of Simulation and Experimental Results}

Experiments are conducted for two different distributions of anchor nodes [11]. A section of roadway are assumed to be $600 \mathrm{~m}$ in length and $5 \mathrm{~m}$ in width .39 unknown nodes are distributed randomly in the two environments and the communication range of the nodes is $120 \mathrm{~m}$. In each environment, the simulation is performed for 10 times and in order to compare the two different anchor nodes layout impact on the final positioning result, the coordinates of unknown nodes in different environment are the same.

Experiments environment 1: in the rectangular area of $600 m \times 5 m, 11$ anchor nodes are distributed on both sides of the roadway evenly and the positions of them are known. The anchor nodes' matrix is as follows: Beacon $=[0: 60: 600 ; 0,5,0,5,0,5,0,5,0,5,0]$. The first line of the matrix is the abscissa of the anchor nodes and the second line is the ordinate. The simulation results obtained and node positioning errors [12] of 39 unknown nodes' matrix generated randomly are shown in figure 5 and Fig. 6.

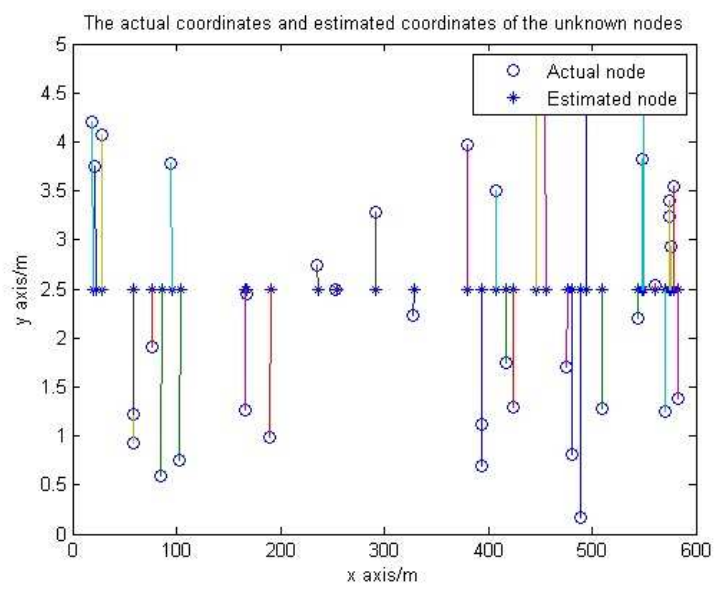

Fig. 5: The simulation results of environment 1

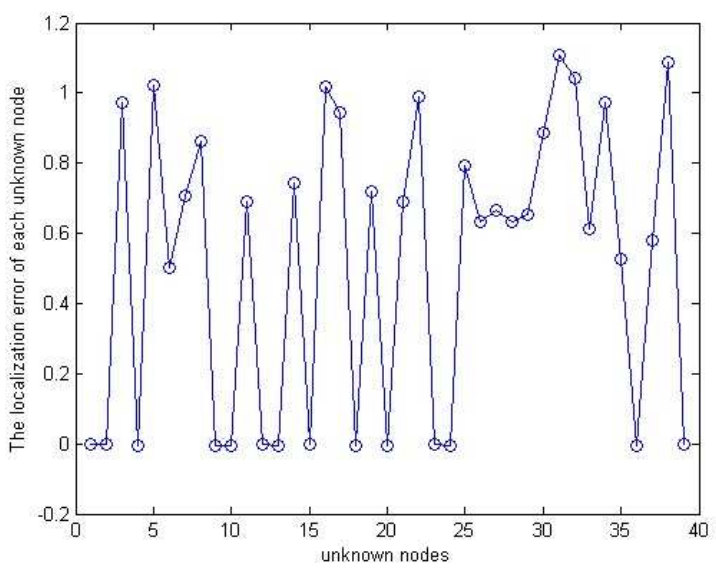

Fig. 6: The node positioning errors of environment 1

Experiments environment 2: in the rectangular area of $600 m \times 5 m, 11$ anchor nodes are distributed on both sides of the roadway evenly and the positions of them are known. The anchor nodes' matrix is as follows: Beacon $=[0: 60: 600 ; 0,2.5,5,2.5,0,2.5,5,2.5,0,2.5,5]$. The first line of the matrix is the abscissa of the anchor nodes and the second line is the ordinate. The 39 unknown nodes' matrix is same as the unknown nodes' matrix in simulation environment 1 for comparing with environment 1 . The simulation results obtained and node positioning errors are shown in Fig. 7 and Fig. 8.

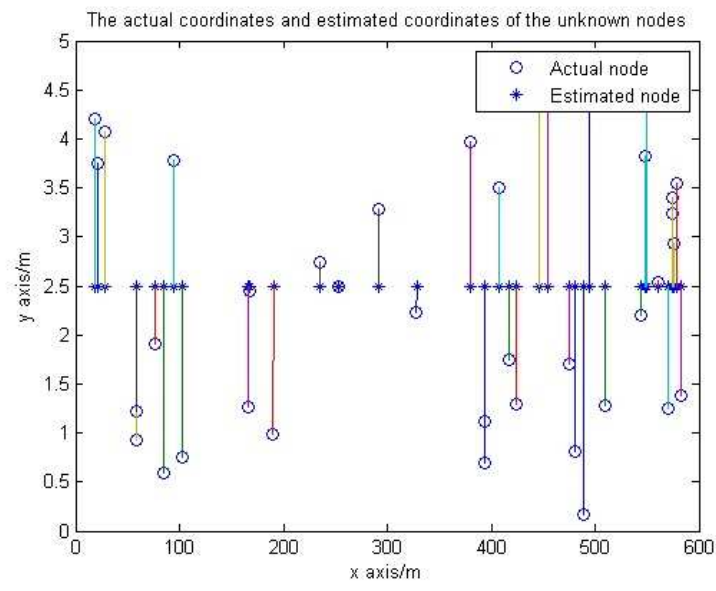

Fig. 7: The simulation results of environment 2

Through the simulation to ten groups of unknown nodes' matrix in two environments, the results obtained are shown in Fig. 9.

By comparing node positioning errors of the anchor nodes laying on both sides and the anchor nodes lying on 


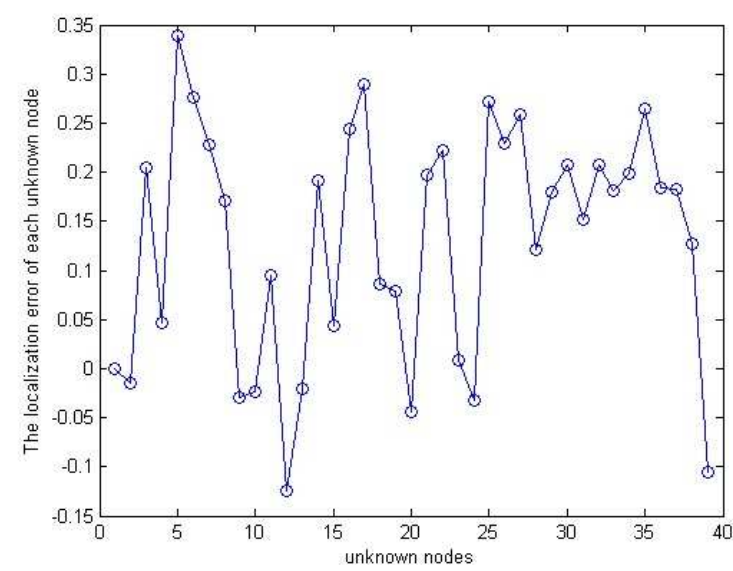

Fig. 8: The node positioning errors of environment 2
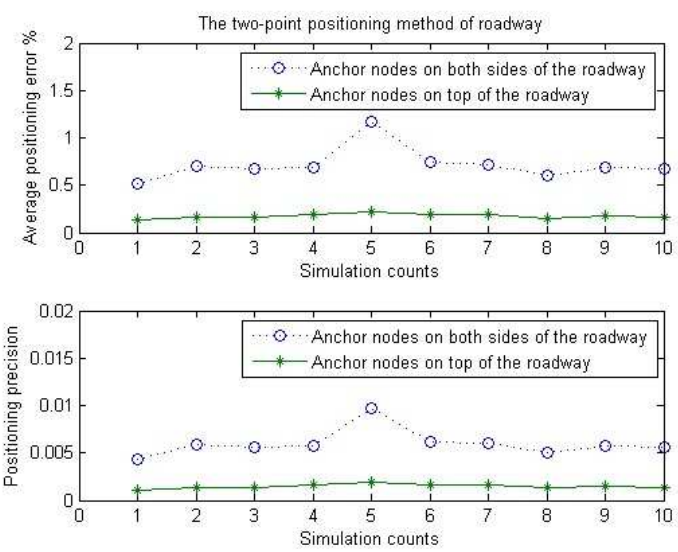

Fig. 9: The simulation comparison diagram

the top of the roadway, the latter has higher positioning accuracy than the former.

\section{Acknowledgement}

This work is supported by national nature science foundation (61170060), Anhui provincial natural science foundation (11040606M135), Anhui provincial natural science foundation of universities key projects (KJ2011A083) , and cooperative project of Huaibei Mining Group Company, "The applied research of the wireless perception system with long distance and low power as well as the mine personnel positioning and hedge".

\section{References}

[1] Jianhong Wang, Shishun Du. Local Area Localization Algorithm for Underground Tunnel Based on WSN. Coal Mine Machinery, 31, 189-191 (2010).

[2] Liang Kong, etc. Positioning Technology Based on RSSI in Coal Mine.Safety in Coal Mines, 43, 48-50 (2012).

[3] He T., Huang C., Blum B., et al. Ranged-free Localization Schemes in Large Scale Sensor Networks. Proc of MOBICOM, 81-95 (2003).

[4] Ryosuke Fujiwara, Kenichi Mizugaki, Tatsuo Nakagawa, et al. TOA/TDOA Hybrid Relative Poistioning System Using UWB-IR. Proceedings of the 4th International Conference on Radio and Wireless Symposium, 647-650 (2009).

[5] Rong Peng, Mihai, Sichitiu. Angle of Arrival Localization for Wireless Sensor Networks. Proc of IEEE SECON, 374-382 (2006).

[6] D. Niculescu, B. Nath. Ad Hoc positioning system(APS). Proc of IEEE Global Telecommunications Conference, 29262931 (2001).

[7] Raghuram, P. Venkatesh, Veeramuthu. Enhancing Mine Safety with Wireless Sensor Networks Using Zigbee Technology. Journal of Theoretical and Applied Information Technology, 37, 261-267 (2012).

[8] Jing Di, etc. Node Localization Algorithm of Wireless Sensor Networks in Coalmine Safety. Computer Simulation, 28, 8891 (2011).

[9] Feng Tian, etc. Nodes Localization Algorithm for Linear Wireless Sensor Network in Underground Coal Mine. Journal of China Coal Society, 35, 1760-1764 (2010).

[10] Holly Moore. MATLAB Practical Guide (Second Edition). Beijing: Publishing House of Electronics Industry, 110-119 (2011).

[11] Takamasa Higuchi, Sae Fujii, Hirozumi Yamaguchi, Teruo Higashino. An Efficient Localization Algorithm Focusing on Stop-and-Go Behavior of Mobile Nodes. IEEE International Conference on Pervasive Computing and Communications (PerCom), 205-212 (2011).

[12] Awad, Frunzke, Dressler. Adaptive Distance Estimation and Localization in WSN Using RSSI Measures. Digital System Design Architectures, Methods and Tools, 471-478 (2007). 


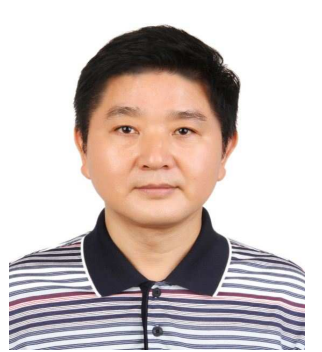

Jingzhao Li received his M.A. degree from China University of Mine and Technology in 1992, and $\mathrm{PhD}$. degree in the key Lab of Power Electronics and Power Drives at Hefei University of Science and Technology, in 2003. He is currently a Professor with the School of Computer Science and Engineering, Anhui University of Science and Technology, China. His research interests include Computer Control, Internet of Things Technology and Embedded Systems. He has published more than 50 papers in domestic and international academic journals and conference proceedings'. These papers are embodied more than 30 times by SCI and EI and are cited more than 50 times by others.

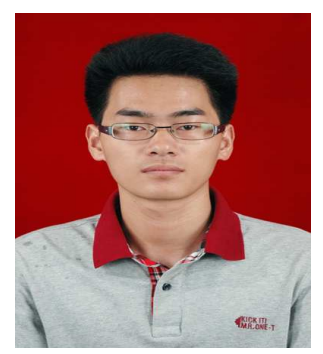

Hong Ma received the B.Eng. degree from Anhui University of Science and Technology, China, in 2011. $\mathrm{He}$ is currently a postgraduate student of computer application technology with the Department of Computer Science and Engineering, Anhui University of Science and Technology, China. His current areas of research are the technology of Internet of things and Embedded technology.

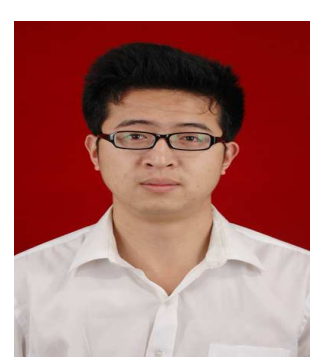

Yubo Cui received his B.Eng.degree from Anhui University of Science and Technology,China,in 2011.His current specialty is computer application technology with the Department of Computer Science and Engineering, Anhui University of Science and Technology, China. His current areas of research are the technology of the Internet of things and Embedded technology. 